\title{
ON THE $K$-THEORY OF LAURENT POLYNOMIALS
}

\author{
S. M. GERSTEN
}

ABstract. The Karoubi-Villamayor $K$-theory of the ring of Laurent polynomials over a regular ring is computed. It is shown that Milnor's $K_{2}$ of a ring of Laurent polynomials over a regular ring maps onto $K_{1}$ of the ring.

In [3] we introduced an algebraic $K$-theory, $\left\{\kappa_{i}^{G \mathrm{~L}}\right\}$, and discussed its general properties. In this article we compute $\kappa_{i}^{\mathrm{GL}}\left(R\left[x, x^{-1}\right]\right)$ for regular rings. More precisely we establish

THEOREM. If $R$ is left regular, then $\kappa_{n+1}^{\mathrm{GL}}\left(R\left[x, x^{-1}\right]\right) \cong \kappa_{n+1}^{\mathrm{GL}}(R)$ $\oplus \kappa_{n}^{\mathrm{GL}}(R)$.

This result should be viewed as a corollary of the techniques of [2] interpreted in the light of the formalism of [3].

As a corollary, we prove that if we write $K_{2}\left(R\left[x, x^{-1}\right]\right)=K_{2}(R) \oplus X$, then if $R$ is left regular, there is a surjection $X \rightarrow K_{1}(R)$. H. Bass has informed me that $\mathrm{J}$. Wagoner also has results about the group $X$ above.

We shall follow the notations of [3] throughout this article. The results will be used in a subsequent paper to study in greater detail the relationship between $K_{2}$ and $\kappa_{2}^{\mathrm{GL}}$.

\section{Polynomial extensions.}

THEOREM 1.1. If $R$ is any ring (without unit) and $R[x]$ is the polynomial ring in one variable $x$, then the inclusion $R \rightarrow R[x]$ induces an isomorphism

$$
\underset{\kappa_{i}}{\mathrm{GL}}(R) \cong \kappa_{i}^{\mathrm{GL}}(R[x])
$$

for all $i \geqq 1$.

Proof. The result is given in $[3, \S 3$, Remark 1$]$. Here is the argument. One considers the s.e.s.

$$
E R \rightarrow R[x] \rightarrow R,
$$

a GL fibration since it splits. The long exact sequence [3, Proposition 2.12 and 5.1], yields

Received by the editors June 5, 1970.

AMS 1970 subject classifications. Primary 13D15, 18F25, 16A54.

$K e y$ words and phrases. Algebraic $K$-theory, Laurent polynomials, loop and path rings, left regular ring. 


$$
\underset{\kappa_{i}}{\mathrm{GL}}(R[x]) \cong \kappa_{i}^{\mathrm{GL}}(R) \oplus \underset{\kappa_{i}}{\mathrm{GL}}(E R) .
$$

Also, by $[3,3.4$ and 5.2$], \kappa_{i}^{\mathrm{GL}}(E R)=0(i \geqq 1)$ from which follows the theorem.

Observe that at the $K_{0}$ level we have

$$
\bar{K}_{0}\left(R[x]^{+}\right)=\bar{K}_{0}\left(R^{+}\right) \oplus \bar{K}_{0}\left(E R^{+}\right) .
$$

If $R$ is left regular (i.e., unital, left noetherian, and each $f g$ module has finite h.d.), then Grothendieck's theorem [2] implies that $\bar{K}_{0}\left(E R^{+}\right)$ $=0$.

For ease of notation, denote $\bar{K}_{0}\left(A^{+}\right)$by $K_{0}(A)$ for any ring $A$. This notation is consistent for if $A$ has a unit, $A^{+} \cong A \pi \mathbf{Z}$ and $K_{0}\left(A^{+}\right)$ $=K_{0}(A) \oplus K_{0}(Z)$.

Proposition 1.2. Let $R$ be a ring and suppose that $R \rightarrow R[X]$ induces an isomorphism $K_{0}(R) \rightarrow K_{0}(R[X])$ for any set $X$, where $R[X]$ is the polynomial ring. Then $K_{0}(E R[X])=0$.

Proposition 1.3. Suppose that $K_{0}(R) \rightarrow K_{0}(R[X])$ is an isomorphism for all $X$. Then $K_{0}(\Omega R) \rightarrow K_{0}(\Omega R[X])$ is an isomorphism.

Proof. Consider the commutative diagram

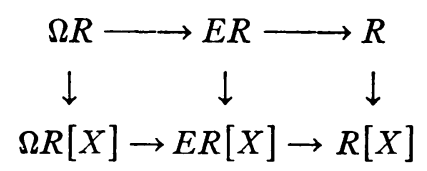

whose rows are s.e.s.'s. By $[3,3.5], E R \rightarrow R$ and $E R[X] \rightarrow R[X]$ are GL-fibrations $((E R)[X]=E(R[X]))$. Hence by $[3,5.10,5.8]$, we have a commutative diagram with exact rows

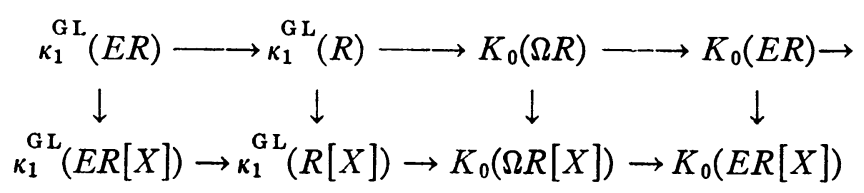

The two left vertical arrows are isomorphisms by 1.1 and the fact that $\kappa_{1}^{G \mathrm{~L}}$ commutes with direct limits. The result follows by 1.2 and the five lemma.

CoROllaRy 1.4. If $K_{0}(R) \rightarrow K_{0}(R[X])$ is an isomorphism for all $X$, then $K_{0}\left(E \Omega^{n} R\right)=0$ for all $n$. In particular this is the case if $R$ is left regular.

COROLLARY 1.5. Under the hypotheses of $1.4, \kappa_{n}^{\mathrm{GL}}(R) \cong K_{0}\left(\Omega^{n} R\right)$. 
Proof. We deduce by "dimension shifting" that $\kappa_{n}^{\mathrm{GL}}(R)$ $\cong \kappa_{1}^{\mathrm{GL}}\left(\Omega^{n-1} R\right)$. The isomorphism $\kappa_{1}^{\mathrm{GL}}\left(\Omega^{n-1} R\right) \cong K_{0}\left(\Omega^{n} R\right)$ follows from 1.4 and the long exact sequence associated to the s.e.s.

$$
\Omega^{n} R \rightarrow E \Omega^{n-1} R \rightarrow \Omega^{n-1} R .
$$

2. Laurent polynomials $R\left[x, x^{-1}\right]$. We pass now to a discussion of $\kappa_{i}^{\mathrm{GL}}\left(R\left[x, x^{-1}\right]\right)$ where $R\left[x, x^{-1}\right]$ is the group ring of the infinite cyclic group generated by $x$. Let us recall the main results of [2].

If $A$ is a unital ring, then there is a group homomorphism

$$
\mathrm{GL}\left(A\left[x, x^{-1}\right]\right) \stackrel{\phi_{A}}{\longrightarrow} K_{0}(A)
$$

which induces a split epimorphism

$$
K_{1}\left(A\left[x, x^{-1}\right]\right) \rightarrow K_{0}(A)
$$

Furthermore $\phi_{A}$ is natural in $A$ in the sense that if $f: A \rightarrow B$ is a unital ring homomorphism, then there is a commutative diagram

$$
\begin{array}{cc}
\mathrm{GL}\left(A\left[x, x^{-1}\right]\right) & \phi_{A} \\
\downarrow & K_{0}(A) \\
\downarrow \mathrm{GL}\left(f\left[x, x^{-1}\right]\right) & \downarrow K_{0}(f) \\
\mathrm{GL}\left(B\left[x, x^{-1}\right]\right) \longrightarrow \phi_{B} & \longrightarrow(B)
\end{array}
$$

In addition, $K_{1}\left(A\left[x, x^{-1}\right]\right) \cong K_{0}(A) \oplus K_{1}(A) \oplus X_{A}$ where $X_{A}$ is generated by unipotents and the decomposition is natural in $A$.

We indicate now the modifications necessary if $R$ is a ring without unit. Recall that $\mathrm{GL}(R)$ is an invariant subgroup of $\mathrm{GL}\left(R^{+}\right)$. We define $\phi_{R}: \operatorname{GL}\left(R\left[x, x^{-1}\right]\right) \rightarrow K_{0}(R)$ to make the following diagram commute

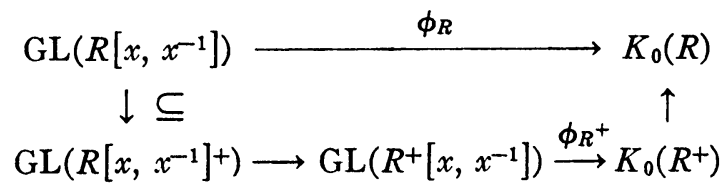

Here, the map $K_{0}\left(R^{+}\right) \rightarrow K_{0}(R)=\bar{K}_{0}\left(R^{+}\right)=\operatorname{Ker}\left(K_{0}\left(R^{+}\right) \rightarrow K_{0}(Z)\right)$ is the obvious retraction. Evidently $\phi_{R}$ so defined is natural in $R$, and this notation is consistent if $R$ has a unit.

We need to determine the precise relationship between $\kappa_{1}^{\mathrm{GL}}\left(R\left[x, x^{-1}\right]^{+}\right)$and $\kappa_{1}^{\mathrm{GL}}\left(R^{+}\left[x, x^{-1}\right]\right)$. Observe that the diagram 


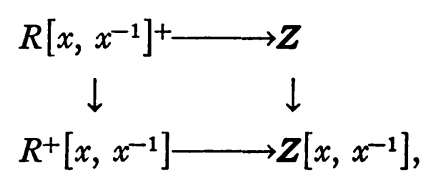

whose horizontal arrows are augmentations and vertical arrows are inclusions, is cartesian, and the horizontal arrows are split. Then the Mayer-Vietoris sequence $[3, \S 2, \S 5]$, becomes

$$
0 \rightarrow \kappa_{1}^{\mathrm{GL}}\left(R\left[x, x^{-1}\right]^{+}\right) \rightarrow \kappa_{1}^{\mathrm{GL}}(Z) \oplus \underset{\kappa_{1}}{\mathrm{GL}}\left(R^{+}\left[x, x^{-1}\right]\right) \rightarrow \kappa_{1}^{\mathrm{GL}}\left(Z\left[x, x^{-1}\right]\right) \rightarrow 0 .
$$

Thus $\kappa_{1}^{\mathrm{GL}}\left(R\left[x, x^{-1}\right]\right)=\operatorname{Ker}\left(\kappa_{1}^{\mathrm{GL}}\left(R^{+}\left[x, x^{-1}\right]\right) \rightarrow \kappa_{1}^{\mathrm{GL}}\left(Z\left[x, x^{-1}\right]\right)\right)$. Since $K_{1}\left(R^{+}\left[x, x^{-1}\right]\right)=K_{1}\left(R^{+}\right) \oplus K_{0}\left(R^{+}\right) \oplus X_{R^{+}}$and since $\kappa_{1}^{\mathrm{GL}}\left(Z\left[x, x^{-1}\right]\right)$ $=K_{1}(Z) \oplus K_{0}(Z)$, if we knew that passage from $K_{1}\left(R^{+}\left[x, x^{-1}\right]\right)$ to $\kappa_{1}^{\mathrm{GL}}\left(R^{+}\left[x, x^{-1}\right]\right)$ did not kill any of the $K_{0}(R)$ factor, we could conclude that

$$
\underset{\kappa_{1}}{\mathrm{GL}}\left(R^{+}\left[x, x^{-1}\right]\right)=\kappa_{1}^{\mathrm{GL}}\left(R^{+}\right) \oplus K_{0}\left(R^{+}\right)
$$

and hence

$$
\underset{\kappa_{1}}{\mathrm{GL}}\left(R\left[x, x^{-1}\right]\right)=\underset{\kappa_{1}}{\mathrm{GL}}(R) \oplus K_{0}(R) .
$$

THEOREM 2.2. Suppose that $R$ is left regular (or more generally that $K_{0}(R) \rightarrow K_{0}(R[X])$ is an isomorphism for all sets $\left.X\right)$. Then for each $n \geqq 0$ the map

$$
\phi_{\Omega^{n} R}: \mathrm{GL}\left(\Omega^{n} R\left[x, x^{-1}\right]\right) \rightarrow K_{0}\left(\Omega^{n} R\right)
$$

factors through $\kappa_{1}^{\mathrm{GL}}\left(\Omega^{n} R\left[x, x^{-1}\right]\right)$. Furthermore, we have

$$
\underset{\kappa_{n+1}}{\mathrm{GL}}\left(R\left[x, x^{-1}\right]\right) \cong \underset{\kappa_{n+1}}{\mathrm{GL}}(R) \oplus \underset{\kappa_{n}}{\mathrm{GL}}(R)
$$

(if $n=0, \kappa_{0}^{\mathrm{GL}}=K_{0}$ ).

Proof. Recall from $[3, \S 5]$, that $\kappa_{1}^{\mathrm{GL}}(R)=\mathrm{GL}(R) / \mathrm{GL}(\partial) \mathrm{GL}(E R)$, $E R=t R[t]$, and $\partial: E R \rightarrow R$ is given by " $t \rightarrow 1$." Furthermore, $E\left(\Omega^{n} R\left[x, x^{-1}\right]\right)=\left(E \Omega^{n} R\right)\left[x, x^{-1}\right]$. Consider now the commutative diagram

$$
\begin{array}{cc}
\mathrm{GL}\left(E \Omega^{n} R\left[x, x^{-1}\right]\right) \stackrel{\phi_{E \Omega^{n} R}}{\longrightarrow} K_{0}\left(E \Omega^{n} R\right) \\
\downarrow \mathrm{GL}(\partial) & \downarrow K_{0}(\partial) \\
\mathrm{GL}\left(\Omega^{n} R\left[x, x^{-1}\right]\right) \stackrel{\phi_{\Omega^{n} R}}{\longrightarrow} & K_{0}\left(\Omega^{n} R\right)
\end{array}
$$


By 1.4, we have $K_{0}\left(E \Omega^{n} R\right)=0$. It follows from the preceding description of $\kappa_{1}^{\mathrm{GL}}$ that $\phi_{\Omega^{n} R}$ factors through $\kappa_{1}^{\mathrm{GL}}\left(\Omega^{n} R\left[x, x^{-1}\right]\right)$.

To establish (2.3), we apply (2.1) to $\Omega^{n} R$ and observe that passage from $K_{1}$ to $\kappa_{1}^{\mathrm{GL}}$ kills all unipotents, but does not disturb the $K_{0}$ factor, by the preceding paragraph. Thus

$$
\kappa_{1}^{\mathrm{GL}}\left(\Omega^{n} R\left[x, x^{-1}\right]\right) \cong \kappa_{1}^{\mathrm{GL}}\left(\Omega^{n} R\right) \oplus K_{0}\left(\Omega^{n} R\right) .
$$

The proof is completed now by "dimension shifting" and by applying 1.5 .

CoROllaRy 2.4. If $R$ is left regular and if we write $K_{2}\left(R\left[x, x^{-1}\right]\right)$ $=K_{2}(R) \oplus X$, then there is a surjection $X \rightarrow K_{1}(R)$.

Proof. The map $\psi: K_{2}\left(R\left[x, x^{-1}\right]\right) \rightarrow \kappa_{2}^{G L}\left(R\left[x, x^{-1}\right]\right)$ of $[3,6.1]$, is surjective since $R\left[x, x^{-1}\right]$ is left regular. Also $\kappa_{2}^{\mathrm{G} L}\left(R\left[x, x^{-1}\right]\right)$ $\cong \kappa_{2}^{\mathrm{GL}}(R) \oplus \kappa_{1}^{\mathrm{GL}}(R)$ by 2.3 . The result follows from the observation that $\kappa_{1}^{\mathrm{GL}}(R)=K_{1}(R)$ if $R$ is left regular, a consequence of $[2$, Theorem 1].

3. $K_{0}$ is not a homotopy functor. This question arises since $\kappa_{i}^{\mathrm{GL}}$ is a homotopy functor for $i \geqq 1$ (see $[3, \S 3]$ ). We observe that

$$
\partial_{x}^{1} \simeq \partial_{x}^{0}: R[x] \rightarrow R
$$

where $\partial_{x}^{i}$ is "evaluation at $i, " i=0,1$. Furthermore, $K_{0}(R[x])=K_{0}(R)$ $\oplus K_{0}(E R)$ where $K_{0}(E R)=\operatorname{Ker} K_{0}\left(\partial_{x}^{0}\right): K_{0}(R[x]) \rightarrow K_{0}(R)$. Thus to show that $K_{0}$ is not a homotopy functor we need only exhibit an element $\eta \in K_{0}(R[x])$ such that $K_{0}\left(\partial_{x}^{0}\right) \eta=0$ but $K_{0}\left(\partial_{x}^{1}\right) \eta \neq 0$. Such an $\eta$ will be a nonzero element of $K_{0}(E R)$.

Let $S$ be the infinite cyclic group generated by $t$, written additively, and make $S$ into a ring by requiring all products be zero. Thus $S=\boldsymbol{Z}[t] t /\left(t^{2}, t^{3}\right)$.

We construct the s.e.s. in Ring of canonical maps

$$
\left(t^{2}, t^{3}\right) Z[t] \rightarrow t Z[t] \rightarrow S,
$$

and let $R=\left(t^{2}, t^{3}\right) \boldsymbol{Z}[t]$. Thus $R^{+}$is a cusp at the origin defined over $\boldsymbol{Z}$. Also let $F=t \boldsymbol{Z}[t]$. We have a commutative diagram with rows s.e.s.'s-

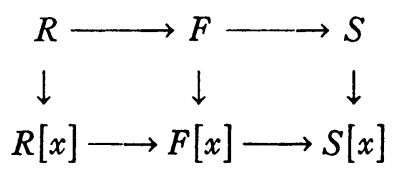

where vertical arrows are inclusions. By results of [2], 


$$
K_{i}(F)=0=K_{i}(F[x])
$$

for $i=0,1 .\left(F^{+}=Z[t], F[x]=E(Z[x])\right.$, and $Z[x]$ is regular.) Thus, the exact sequence of $[3,5.8]$, yields a commutative diagram

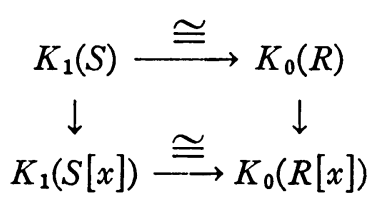

where horizontal arrows are isomorphisms. Thus, to construct an $\eta \in K_{0}(R[x])$ having the required properties, it is equivalent to construct $\xi \in K_{1}(S[x])$ such that $K_{1}\left(\partial_{x}^{0}\right) \xi=0, K_{1}\left(\partial_{x}^{1}\right) \xi \neq 0 ; \partial_{x}^{i}: S[x] \rightarrow S$ is evaluation at $i, i=0,1$.

Let $\xi$ be the class of the unit $1+t x$ of $S[x]+$ in $K_{1}(S[x])$. Using the determinant it is very easy now to verify that $\xi$ has the required properties.

We have observed already that $\eta$ is a nonzero element of $K_{0}(E R)$ such that $K_{0}\left(\partial_{x}^{1}\right) \eta \neq 0$. Consider now the commutative diagram of $\S 2$ :

$$
\begin{array}{cc}
\mathrm{GL}\left(E R\left[x, x^{-1}\right]\right) \stackrel{\phi_{E R}}{\longrightarrow} K_{0}(E R) \\
\downarrow \mathrm{GL}\left(\partial_{x}^{1}\right) & \quad \downarrow K_{0}\left(\partial_{x}^{1}\right) \\
\mathrm{GL}\left(R\left[x, x^{-1}\right]\right) \stackrel{\phi_{R}}{\longrightarrow} K_{0}(R)
\end{array}
$$

Since $\phi_{E R}$ is surjective, it follows that the composition $\phi_{R} \circ \operatorname{GL}\left(\partial_{x}^{1}\right)$ $\neq 0$. But we saw that

$$
\underset{\kappa_{1}}{\mathrm{GL}}\left(R\left[x, x^{-1}\right]\right)=\mathrm{GL}\left(R\left[x, x^{-1}\right]\right) / \operatorname{Im} \mathrm{GL}\left(\partial_{x}^{1}\right) .
$$

It follows that $\phi_{R}$ does not factor through $\kappa_{1}^{G L}$ for $R=\left(t^{2}, t^{3}\right) Z[t]$.

\section{REFERENCES}

1. H. Bass, Algebraic K-theory, Benjamin, New York, 1968.

2. H. Bass, A. Heller and R. G. Swan, The Whitehead group of a polynomial extension, Inst. Haute Etudes Sci. Publ. Math. No. 22 (1964), 61-79. MR 30 \#4806.

3. S. M. Gersten, On Mayer-Vietoris functors and algebraic $K$-theory, J. Algebra 18 (1971).

4. M. Karoubi and O. Villamayor, Foncteurs $K^{n}$ en algèbre et en topologie, C. R. Acad. Sci. Paris Sér. A-B 269 (1969), 416-419.

Rice University, Houston, TeXas 77001 